\title{
AMPLIFICATION PATH LENGTH DEPENDENCE STUDIES OF STIMULATED EMISSION FROM OPTICALLY PUMPED InGaN/GaN MULTIPLE QUANTUM WELLS
}

\author{
T.J. Schmidt,* S. Bidnyk, * Yong-Hoon Cho, ${ }^{*}$ A.J. Fischer,* J.J. Song, * S. Keller,** \\ U.K. Mishra,** and S.P. DenBaars** \\ *Center for Laser and Photonics Research and Dept. of Physics \\ Oklahoma State University, Stillwater, OK 74078 \\ **Electrical and Computer Engineering and Materials Depts. \\ University of California, Santa Barbara, CA 93106
}

Cite this article as: MRS Internet J. Nitride Semicond. Res. 4S1, G6.54 (1999)

\begin{abstract}
Optically pumped stimulated emission (SE) from InGaN/GaN multiple quantum wells (MQWs) grown by metalorganic chemical vapor deposition has been systematically studied as a function of excitation length $\left(\mathrm{L}_{\mathrm{exc}}\right)$. Two distinct SE peaks were observed from these structures: one that originates at $425 \mathrm{~nm}$ at $10 \mathrm{~K}(430 \mathrm{~nm}$ at $300 \mathrm{~K})$ and another that originates at $434 \mathrm{~nm}$ at $10 \mathrm{~K}(438 \mathrm{~nm}$ at $300 \mathrm{~K})$. The $\mathrm{SE}$ threshold for the high energy peak was observed to always be lower than that of the low energy peak, but the difference was found to decrease greatly with increasing $\mathrm{L}_{\mathrm{exc}}$. A detailed study of the emission intensity of these two SE peaks as a function of excitation density shows that the two peaks compete for gain in the MQW active region.
\end{abstract}

\section{INTRODUCTION}

GaN-based semiconductors and related heterostructures are attracting an ever-increasing amount of attention due to their large direct band gaps, which make them promising materials for UV-blue-green light emitting devices, ${ }^{1,2}$ solar-blind UV detectors, ${ }^{3}$ and high power and high temperature devices. $^{4,5}$ In particular, the realization of high brightness blue and green light emitting diodes ${ }^{1}$ and $\mathrm{cw}$ blue laser diodes ${ }^{2}$ based on InGaN/GaN multiple quantum wells (MQWs) has focused the efforts of many research groups on these structures. Although a considerable amount of research has been conducted on the optical properties of these materials, there is still much left unknown about the optical processes associated with stimulated emission (SE) and lasing. To aid in a fundamental understanding of the processes leading to optical gain in these structures, excitation length dependent studies of optically pumped SE have been performed and illustrate dramatically different SE behavior for changes in the experimental conditions that would typically be insignificant in other semiconductor materials.

Recently, a considerable amount of attention has been given to the potential role of strongly localized band tail states on the SE and lasing processes in InGaN MQWs. ${ }^{6-11}$ Although there exists a sizable amount of data to support localized carrier recombination as the mechanism leading to spontaneous emission in these materials, ${ }^{8,9,12,13}$ the results for SE behavior in the literature are varied and often contradictory. This has led some research groups to assign the spontaneous emission peak to recombination of localized carriers, and the SE peak to a more traditional recombination mechanism: that of an electron-hole plasma originating from free

carriers, ${ }^{14}$ while others claim that strongly localized carriers are the origin of both spontaneous and SE. ${ }^{9-11}$ With the recent observation of two different SE peaks from InGaN/GaN MQWs 
grown by Nichia Chemical Industries ${ }^{11}$ we see the possibility that some of the varied results reported in the literature may stem from slightly different experimental conditions, which are shown here to result in significant changes in the SE behavior. We report the results of a detailed study of the SE behavior of these two SE peaks as a function of excitation length $\left(\mathrm{L}_{\mathrm{exc}}\right)$ and excitation density $\left(\mathrm{I}_{\mathrm{exc}}\right)$ and illustrate dramatically different SE behavior in InGaN MQWs for relatively small changes in the experimental conditions. The observation of these two distinct SE peaks from InGaN/GaN MQWs grown under different conditions by separate research groups suggests this SE behavior is a general property of present state-of-the-art InGaN based blue laser diodes. As such, a better understanding of the SE and lasing behavior of these structures is important for the development and optimization of future laser diode structures.

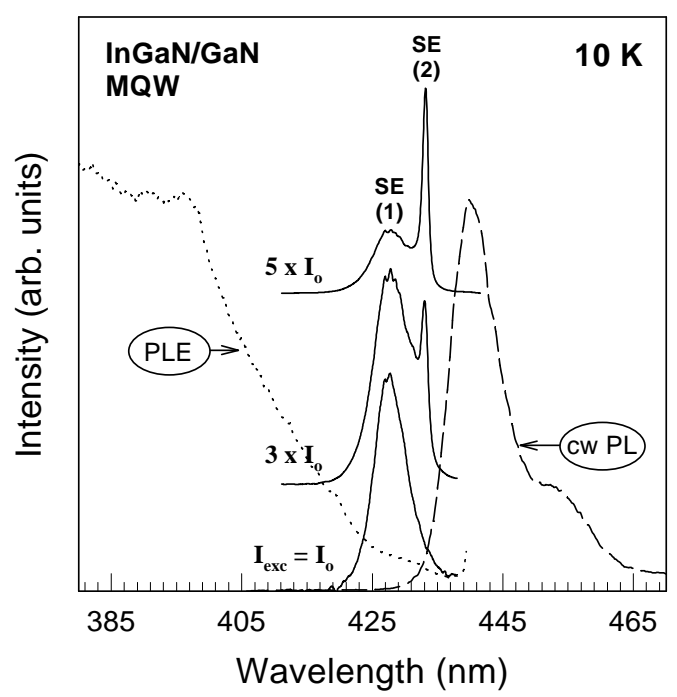

Figure 1: $10 \mathrm{~K}$ stimulated emission spectra (solid lines) from an InGaN/GaN MQW subjected to several excitation densities, where $I_{o}=100 \mathrm{~kW} / \mathrm{cm}^{2}$. The low power PL (dashed line) and PLE (dotted line) spectra are also shown for comparison. The SE spectra have been normalized and displaced vertically for clarity.

\section{EXPERIMENT}

The InGaN/GaN MQWs used in this study were grown by metalorganic chemical vapor deposition (MOCVD) on $1.8 \mu \mathrm{m}$ thick GaN buffer layers grown on (0001) oriented sapphire substrates. The active regions were made up of 12 quantum wells consisting of $3 \mathrm{~nm}$ thick $\mathrm{In}_{0.2} \mathrm{Ga}_{0.8} \mathrm{~N}$ wells and $4.5 \mathrm{~nm}$ thick GaN barriers. The structures were capped by $0.1 \mu \mathrm{m}$ thick $\mathrm{Al}_{0.07} \mathrm{Ga}_{0.93} \mathrm{~N}$ layers. A detailed description of the growth conditions is given elsewhere. ${ }^{15}$ The InGaN MQWs were optically pumped by the third harmonic of an injection seeded Nd:YAG laser $(355 \mathrm{~nm}, 30 \mathrm{~Hz}, \sim 6 \mathrm{~ns}$ pulse width). The excitation beam was focused to a line on the sample using a cylindrical lens and the excitation length was varied using a mask connected to a computer controlled stepper motor. The emission was collected from one edge of the sample, coupled into a 1-meter spectrometer, and spectrally analyzed using an optical multi-channel analyzer.

\section{RESULTS}

Typical power dependent emission spectra at $10 \mathrm{~K}$ are shown in Fig. 1 for $\mathrm{L}_{\mathrm{exc}}=1300 \mu \mathrm{m}$. At low $\mathrm{I}_{\mathrm{exc}}$, we observe a broad spontaneous emission peak centered at $\sim 441 \mathrm{~nm}$, consistent with low power cw photoluminescence (PL) spectra. As $\mathrm{I}_{\text {exc }}$ is increased, a new peak emerges at $\sim 428 \mathrm{~nm}$ [designated here as SE peak (1)] and grows superlinearly with increasing $\mathrm{I}_{\text {exc }}$. If we continue to increase $\mathrm{I}_{\mathrm{exc}}$, we observe another new peak at $\sim 433 \mathrm{~nm}$ [designated here as SE peak (2)] which also grows superlinearly with increasing $\mathrm{I}_{\text {exc. }}$ SE peak (1) is observed to be the statistical distribution of a multitude of narrow $(<0.1 \mathrm{~nm})$ emission lines. No significant broadening of these emission lines was observed as the temperature was tuned from $10 \mathrm{~K}$ to over $500 \mathrm{~K}$. This is illustrated in Fig. 2. SE peak (2) is observed to always be considerably narrower than SE peak (1). Both SE peaks are seen to originate on the high energy 


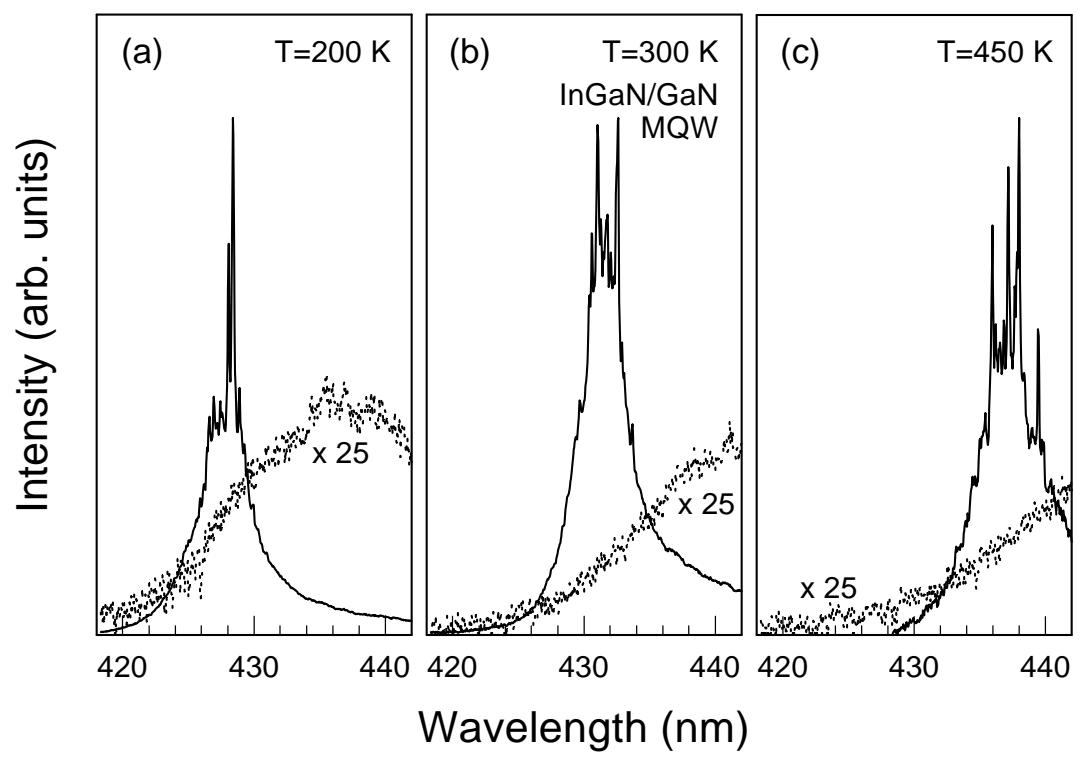

Figure 2: Stimulated emission spectra of SE peak (1) at (a) $200 \mathrm{~K}$, (b) $300 \mathrm{~K}$, and (c) $450 \mathrm{~K}$ illustrating SE peak (1) is composed of a multitude of narrow $(<0.1 \mathrm{~nm})$ stimulated emission peaks that do not noticeably broaden with increasing temperature. The SE spectra shown were collected for excitation densities twice the SE threshold for the respective temperatures. The spontaneous emission spectra (dotted lines) are also shown for excitation densities half that of the SE threshold at each temperature. The SE spectra have been normalized for clarity.

side of the low power spontaneous emission peak (given by the dashed line in Fig. 1) and are seen to be redshifted by more than $30 \mathrm{~nm}$ below the start of the "soft" absorption edge. Both SE peaks were found to be highly TE polarized, with a TE to TM ratio of 200. SE peak (2) has been the subject of previous experiments performed by the authors and has been attributed to stimulated recombination of localized states through the use of energy selective optically pumped SE studies showing "mobility edge" type behavior in the SE spectra as the excitation photon energy is varied, ${ }^{16}$ and through nanosecond nondegenerate optical pump-probe experiments showing the onset of SE has a direct impact on the bleaching dynamics of the band tail states in these samples. ${ }^{17}$

Figure 3(a) shows the SE thresholds ( $\left.\mathrm{I}_{\mathrm{th}}\right)$ of SE peaks (1) and (2) as a function of $\mathrm{L}_{\mathrm{exc}}$. We note that $I_{\text {th }}$ for peak (2) is larger than that of peak (1) for all excitation lengths employed, but approaches that of peak (1) with increasing $\mathrm{L}_{\mathrm{exc}}$. The high $\mathrm{SE}$ threshold of peak (2) with respect to peak (1) and its increased presence for longer $L_{\text {exc }}$ suggest that it results from a lower gain process than that of peak (1). Fig. 3(b) shows the peak positions of SE peak (1) and SE peak (2) as a function of $\mathrm{L}_{\mathrm{exc}}$ at $10 \mathrm{~K}$. For $\mathrm{L}_{\mathrm{exc}}$ less than $\sim 500 \mu \mathrm{m}$, only SE peak (1) is observed. It has a peak emission wavelength of $\sim 425 \mathrm{~nm}(430 \mathrm{~nm})$ and a SE threshold of $\sim 100 \mathrm{~kW} / \mathrm{cm}^{2}(475$ $\left.\mathrm{kW} / \mathrm{cm}^{2}\right)$ at $10 \mathrm{~K}(300 \mathrm{~K})$. As $\mathrm{I}_{\text {exc }}$ is increased and/or $\mathrm{L}_{\mathrm{exc}}$ is increased, a new SE peak [SE peak (2)] at $434 \mathrm{~nm}$ (438 $\mathrm{nm}$ at RT) emerges. The peak positions were measured for $\mathrm{I}_{\text {exc }}$ fixed relative to the SE thresholds of the respective peaks; i.e. $\mathrm{I}_{\mathrm{exc}}=2 \times \mathrm{I}_{\mathrm{th}}$. As $\mathrm{L}_{\mathrm{exc}}$ is increased, SE peak (1) shifts to longer wavelengths (due to a re-absorption process), while the peak position of SE peak (2) is observed to be weakly dependent on $\mathrm{L}_{\text {exc }}$. The apparent blueshift of SE peak (2) with increasing $\mathrm{L}_{\mathrm{exc}}$ seen in Fig. 3(b) is a result of the experimental conditions. Since the SE threshold of SE peak (2) is a strong function of $\mathrm{L}_{\mathrm{exc}}$, the peak positions shown for small $\mathrm{L}_{\mathrm{exc}}$ are for $\mathrm{I}_{\mathrm{exc}}$ considerably higher than for large $\mathrm{L}_{\mathrm{exc}}$. The slight redshift of SE peak (2) with increasing $\mathrm{I}_{\mathrm{exc}}$ due to many-body effects and lattice heating then manifests itself as the apparent blueshift seen in Fig. 3(b). The same phenomenon is observed at room temperature, as shown in the inset of Fig. 3(b). The redshift of SE peak (1) with increasing $\mathrm{L}_{\mathrm{exc}}$ can be explained by gain and 

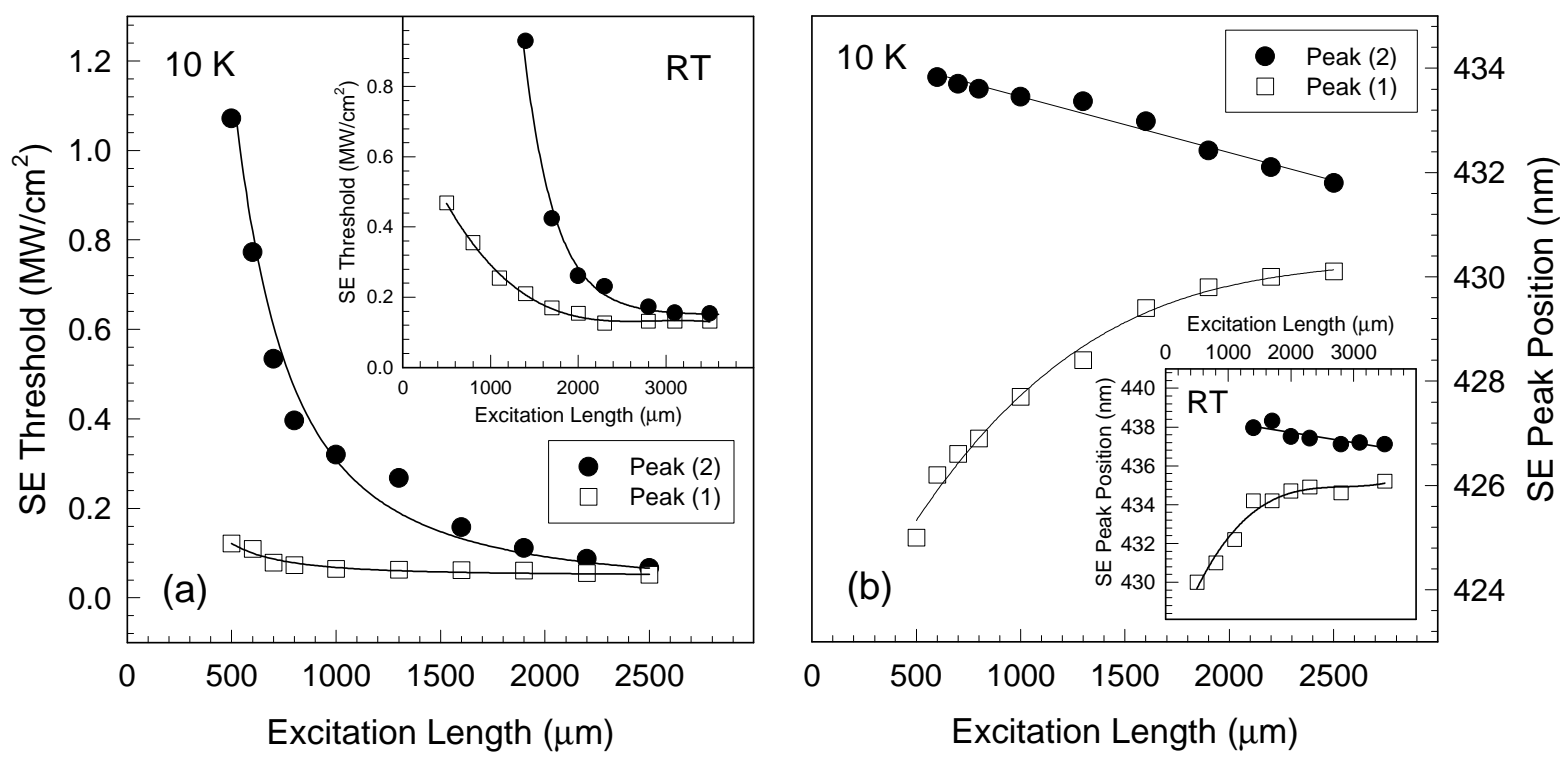

Figure 3: (a) Stimulated emission threshold as a function of excitation length for SE peaks (1) and (2) at $10 \mathrm{~K}$. (b) Peak position of SE peaks (1) and (2) as a function of excitation length at $10 \mathrm{~K}$. The insets show the behavior observed at room temperature. The solid lines are given only as guides for the eye.

absorption competition in the "soft" absorption edge of the InGaN active regions, where gain saturation with longer $\mathrm{L}_{\mathrm{exc}}$ combined with the background absorption tail leads to the observed redshift. The fact that SE peak (2) does not experience a re-absorption induced redshift with increasing $L_{e x c}$ is explained by the significant reduction of the absorption tail in this spectral region (see Fig. 1).

The gain saturation behavior of SE peak (1) is consistent with the observation of Kuball et al. ${ }^{18}$ of a high gain mechanism in the band tail region of MQWs with similar active regions. The large spectral range exhibiting gain is explained by compositional fluctuations inside the active region. The redshift of SE peak (1) with increasing $\mathrm{L}_{\mathrm{exc}}$ is consistent with observations of a redshift in the optical gain spectrum with increasing $\mathrm{L}_{\text {exc }}$ reported by Mohs et al. ${ }^{19}$ It is also consistent with the observation by Nakamura ${ }^{20}$ that the external quantum efficiency of his cw blue laser diodes decreases with increasing cavity length. These similarities, combined with the relatively low SE threshold of SE peak (1) with respect to SE peak (2) and its similar spectral position with laser emission from diodes of similar structure, ${ }^{21}$ suggests that lasing in current state-of-the-art $\mathrm{cw}$ blue laser diodes originates from the gain mechanism responsible for SE peak (1). The microscopic origin of this

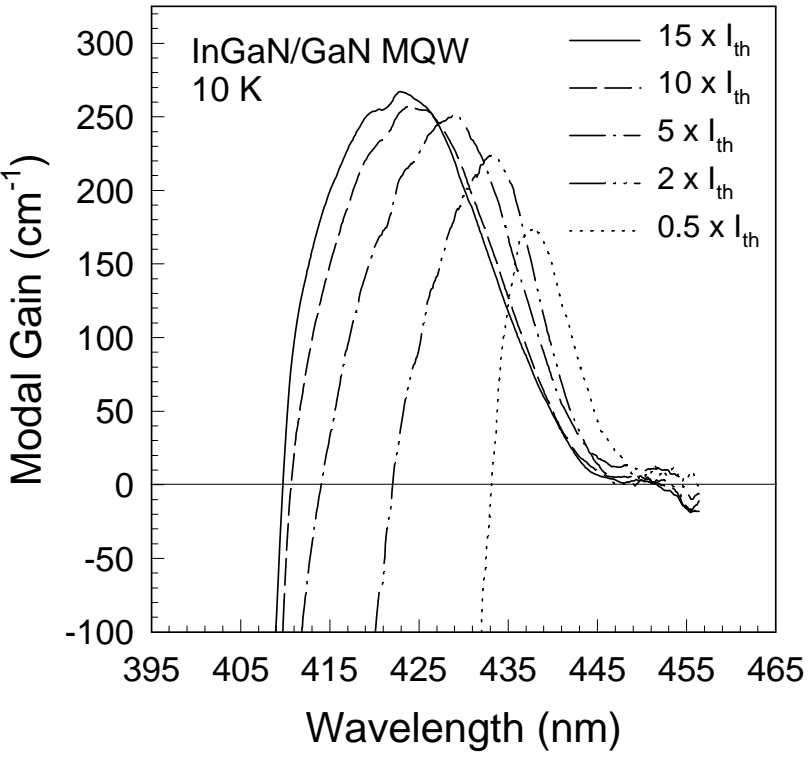

Figure 4: $10 \mathrm{~K}$ modal gain spectra of an $\mathrm{InGaN} / \mathrm{GaN} \mathrm{MQW}$ at several excitation densities. The excitation densities are given with respect to the SE threshold measured for long $(\sim 2.5 \mathrm{~mm})$ excitation lengths. 
gain is the subject of continuing research, but is shown here to be different than the localized state recombination responsible for $\mathrm{SE}$ peak (2). Its origin may lie in an entirely different degree of carrier localization. Further experiments are needed to clarify this issue.

The modal gain spectra measured by the variable-stripe method of Shaklee and Leheny ${ }^{22}$ are shown in Fig. 4 at $10 \mathrm{~K}$. The spectra were taken for $\mathrm{L}_{\mathrm{exc}}$ less than $200 \mu \mathrm{m}$ to minimize re-absorption induced distortions in the spectra. The excitation densities in Fig. 4 are given with respect to the $\mathrm{SE}$ threshold measured for long ( $2.5 \mathrm{~mm})$ excitation lengths. A clear blueshift in the gain peak is seen with increasing excitation.

This blueshift was observed to stop for $\mathrm{I}_{\mathrm{exc}}>12 \mathrm{x} \mathrm{I}_{\text {th }}$. Further increases in $\mathrm{I}_{\mathrm{exc}}$ resulted only in an increase in the modal gain maximum. The maxima of the gain spectra are seen to be redshifted by more than $20 \mathrm{~nm}$ with respect to the start of the "soft" absorption edge. The large shift in the gain maximum to higher energy with increasing $\mathrm{I}_{\mathrm{exc}}$ is consistent with band filling of localized states in the InGaN active regions. We note that the fully blueshifted gain spectrum covers the entire spectral region in which SE was observed. Similar behavior was observed at room temperature.

The dependence of the emission intensity of peaks (1) and (2) on $\mathrm{I}_{\mathrm{exc}}$ is shown in Fig. 5 for $\mathrm{L}_{\mathrm{exc}}=1300 \mu \mathrm{m}$ at $10 \mathrm{~K}$. The emission of peak (1) increases in a strongly superlinear fashion $\left(\sim \mathrm{I}_{\text {exc }}{ }^{3.8}\right)$ until the SE threshold of peak (2) is reached, at which point it turns linear, indicating that peak (2) competes for gain with peak (1). This is most likely a result of competition for carriers or re-absorption of the emitted photons. The presence of SE peak (2) is therefore seen to be deleterious to SE peak (1). The same process is observed at room temperature and for various excitation lengths. This gain competition may limit this material's performance in high power laser diode applications, where increased driving current and/or longer cavity lengths may result in a shift in the dominant gain mechanism and a drastic change in the emission behavior.

\section{CONCLUSIONS}

In summary, the dependence of stimulated emission on excitation length in MOCVD grown InGaN/GaN MQW structures has been studied. Two distinctly different SE peaks were observed with different dependencies on excitation length. The high energy SE peak exhibits a strong redshift with increasing excitation length due to competition between an easily saturable gain mechanism and a background absorption tail. The lower energy SE peak does not exhibit this re-absorption induced redshift with increasing excitation length. The presence of the lower energy SE peak has been shown to be detrimental to the higher energy SE peak due to gain competition in the InGaN active region. This competition may prove to be an obstacle in the 
design of InGaN based high power laser diodes, where high current densities and/or long cavity lengths can lead to a shift in the dominant gain mechanism and a change in the emission characteristics.

\section{ACKNOWLEDGEMENTS}

This work was supported by BMDO, AFOSR, ARO, ONR, and DARPA.

\section{REFERENCES}

${ }^{1}$ S. Nakamura, M. Senoh, N. Iwasa, S. Nagahama, T. Yamada, and T. Mukai, Jpn. J. Appl. Phys. 34, L1332 (1995).

${ }^{2}$ S. Nakamura, M. Senoh, S. Nagahama, N. Iwasa, T. Yamada, T. Matsushita, Y. Sugimoto, and H. Kiyoku, Appl. Phys. Lett. 69, 4056 (1996).

${ }^{3}$ B.W. Lim, Q.C. Chen, J.Y. Yang, and M.A. Khan, Appl. Phys. Lett. 68, 3761 (1996).

${ }^{4}$ Y.F. Wu, B.P. Keller, S. Keller, D. Kapolnek, P. Kozodoy, S.P. DenBaars, and U.K. Mishra, Appl. Phys. Lett. 69, 1438 (1996).

${ }^{5}$ X.H. Yang, T.J. Schmidt, W. Shan, J.J. Song, and B. Goldenberg, Appl. Phys. Lett. 66, 1 (1995). S. Bidnyk, T.J. Schmidt, Y.H. Cho, G.H. Gainer, J.J. Song, S. Keller, U.K. Mishra, and S.P. DenBaars, Appl. Phys. Lett. 72, 1623 (1998).

${ }^{6}$ S. Nakamura, M. Senoh, S.I. Nagahama, N. Iwasa, T. Yamada, T. Matsushita, Y. Sugimoto, and H. Kiyoku, Appl. Phys. Lett. 70, 1417 (1997).

${ }^{7}$ S. Nakamura, M. Senoh, S.I. Nagahama, N. Iwasa, T. Yamada, T. Matsushita, Y. Sugimoto, and H. Kiyoku, Appl. Phys. Lett. 70, 2753 (1997).

${ }^{8}$ S. Chichibu, T. Azuhata, T. Sota, and S. Nakamura, Appl. Phys. Lett. 69, 4188 (1996).

${ }^{9}$ Y. Narukawa, Y. Kawakami, M. Funato, S. Fujita, S. Fugita, and S. Nakamura, Appl. Phys. Lett. 70, 981 (1997).

${ }^{10}$ A. Satake, Y. Masumoto, T. Miyajima, T. Asatsuma, F. Nakamura, and M. Ikeda, Phys. Rev. B 57, R2041 (1998).

${ }^{11}$ T. Deguchi, T. Azuhata, T. Sota, S. Chichibu, M. Arita, H. Nakanishi, and S. Nakamura, Semicond. Sci. Technol. 13, 97 (1998).

${ }^{12}$ E.S. Jeon, V. Kozlov, Y.K. Song, A Vertikov, M. Kuball, A.V. Nurmikko, H. Liu, C. Chen, R.S. Kern, C.P. Kuo, and M.G. Craford, Appl. Phys. Lett. 69, 4194 (1996).

${ }^{13}$ Y. Narakawa, Y. Kuwakima, S. Fujita, S. Fujita, and S. Nakamura, Phys. Rev. B 55, R1938 (1997).

${ }^{14}$ J.S. Im, S. Heppel, H. Kollmer, A. Sohmer, J. Off, F. Scholz, and A. Hangleiter, Proc. $2^{\text {nd }}$ Int. Conf. Nitride Semicond., Tokushima, Japan, 228 (1997).

${ }^{15}$ S. Keller, A.C. Abare, M.S. Minsky, X.H. Wu, M.P. Mack, J.S. Speck, E. Hu, L.A. Coldren, U.K. Mishra, and S.P. DenBaars, Materials Science Forum 264-268, 1157 (1998). B.P. Keller, S. Keller, D. Kapolnek, W.N. Jiang, X.-F. Wu, H. Masui, X.H. Wu, B. Heying, J.S. Speck, U.K. Mishra, and S.P. DenBaars, J. Electron. Mater. 24, 1707 (1995).

16 T.J. Schmidt, Y.H. Cho, G.H. Gainer, J.J. Song, S. Keller, U.K. Mishra, and S.P. DenBaars, Appl. Phys. Lett. 73, 560 (1998).

17 T.J. Schmidt, Y.H. Cho, G.H. Gainer, J.J. Song, S. Keller, U.K. Mishra, and S.P. DenBaars, Appl. Phys. Lett. 73, 1892 (1998).

${ }^{18}$ M. Kuball, E.S. Jeon, Y.K. Song, A.V. Nurmikko, P. Kozodoy, A. Abare, S. Keller, L.A. Coldren, U.K. Mishra, S.P. DenBaars, and D.A. Steigerwald, Appl. Phys. Lett. 70, 2580 (1997).

${ }^{19}$ G. Mohs, T. Aoki, M. Nagai, R. Shimano, M. Kuwata-Gonokami, and S. Nakamura, Proc. $2^{\text {nd }}$ Int. Conf. Nitride Semicond., Tokushima, Japan, 234 (1997).

${ }^{20}$ S. Nakamura, MRS Internet J. Nitride Semicond. Res. 2, Art. 5 (1997).

${ }^{21}$ M.P. Mack, A. Abare, M. Aizcorbe, P. Kozodoy, S. Keller, U.K. Mishra, L. Coldren, and S.P. DenBaars, MRS Internet J. Nitride Semicond. Res. 2, Art. 41 (1997).

${ }^{22}$ K.L. Shaklee and R.F. Leheny, Appl. Phys. Lett. 18, 475 (1971). 